\title{
Vivências de luto e saúde mental da enfermagem na pandemia da COVID-19: o que nos diz a literatura?
}

\author{
Impacts of mourning experience on pandemic COVID-19 and mental health of nursing team?
}

¿Impactos de la experiencia de luto en la pandemia de la COVID-19 y en la salud mental del equipo de enfermería?

\begin{abstract}
RESUMO
Objetivo: mapear as evidências existentes na literatura sobre os lutos vividos pelos profissionais de enfermagem durante a pandemia da Covid-19 e discutir sobre as repercussões para sua saúde mental. Método: revisão integrativa realizada nas bases de dados Pubmed, Cinahl, Scopus e Lilacs, alcançando um total de 198 publicações. Após aplicação dos critérios de inclusão e exclusão, obteve-se um corpus de 04 artigos. Resultados: Os estudos analisados foram realizados no Brasil e no Reino Unido. Dois estudos investigaram aspectos da saúde mental no enfrentamento da Covid-19 específicos para a equipe de enfermagem, um abordou todos os profissionais de saúde e o outro pesquisou os profissionais de saúde e a população em geral. Conclusão: a convivência com situações de luto faz emergir sentimentos que repercutem na saúde mental dos profissionais de enfermagem. Destaca-se a necessidade de mais estudos que fomentem ações de apoio a vivência de luto na pandemia.
\end{abstract}

DESCRITORES: Equipe de enfermagem; Luto; Saúde mental; COVID-19.

\section{ABSTRACT}

Objective: to map the existing evidence in the literature on the grief experienced by nursing professionals during the Covid-19 pandemic and to discuss the repercussions for their mental health. Method: an integrative review perfomed in the Pubmed, Cinahl, Scopus and Lilacs databases, reaching a total of 198 publications. After applying the inclusion and exclusion criteria, a corpus of 04 articles was obtained. Results: The analyzed studies were carried out in Brazil and the United Kingdom. Two studies investigated aspects of mental health in coping with Covid-19 specific to the nursing team, one addressed all health professionals and the other surveyed health professionals and the general population. Conclusion: living with situations of mourning gives rise to feelings that affect the mental health of nursing professionals. The need for further studies to promote actions to support the experience of mourning in the pandemic is highlighted.

DESCRIPTORS: Nursing, Team; Bereavement; Mental Health; COVID-19.

\section{RESUMEN}

Objetivo: mapear la evidencia existente en la literatura sobre el duelo experimentado por los profesionales de enfermería durante la pandemia Covid-19 y discutir las repercusiones para su salud mental. Método: revisión integradora realizada en las bases de datos Pubmed, Cinahl, Scopus y Lilacs, alcanzando un total de 198 publicaciones. Luego de aplicar los criterios de inclusión y exclusión, se obtuvo un corpus de 04 artículos. Resultados: Los estudios analizados se realizaron en Brasil y Reino Unido. Dos estudios investigaron aspectos de la salud mental en el afrontamiento de Covid-19 específicos del equipo de enfermería, uno se dirigió a todos los profesionales de la salud y el otro a los profesionales de la salud encuestados y a la población en general. Conclusión: convivir con situaciones de duelo genera sentimientos que afectan la salud mental de los profesionales de enfermería. Se destaca la necesidad de realizar más estudios para promover acciones que apoyen la experiencia del duelo en la pandemia. DESCRIPTORES: Grupo de Enfermería; Aflicción; Salud Mental; COVID-19.

RECEBIDO EM: 28/11/2020 APROVADO EM: 24/12/2020

\section{Kalyne Patricia de Macedo Rocha}

Graduanda em Enfermagem. Estudante universitária, Universidade Federal do Rio Grande do Norte.

ORCID: 0000-0002-8557-1616

\section{Wanessa Cristina Tomaz dos Santos Barros}

Enfermeira, Doutora em Enfermagem. Professora Universitária, , Universidade Federal do Rio Grande do Norte.

ORCID:0000-0002-1924-3278 


\section{Luciane Paula B. Araújo de Oliveira}

Enfermeira, Doutora em Enfermagem. Professora Universitária, Universidade Federal do Rio Grande do Norte. ORCID:0000-0003-1629-8991

\section{José Jailson de Almeida Júnior}

Enfermeiro, Doutor em Educação. Professor Universitário, Universidade Federal do Rio Grande do Norte. ORCID: 0000-0001-7448-0703

\section{INTRODUÇÃO}

$\square$ m março de 2020 a Organização Mundial de Saúde (OMS) declarou a Pandemia de Covid-19. Desde então, em todo o mundo, enfermeiros e técnicos de enfermagem são homenageados pelo trabalho e compromisso na prestação de cuidados. No entanto, essa vivência tem sido permeada por uma gama de experiências que geram medo, insegurança, perdas e sofrimento ${ }^{1}$.

A pandemia trouxe consigo preocupações com o abastecimento de equipamentos de proteção individual; possibilidade de inabilidade com a nova doença e também com novos setores de trabalho; falta de conhecimento sobre formas de contágio e medicamentos específicos; escassez de leitos de Unidade de Terapia Intensiva e ventiladores mecânicos; sobrecarga de trabalho e a mais desafiadora de todas as questões: o desconhecimento sobre uma doença perigosa que atingiu de maneira letal milhares de profissionais de saúde em todo o mundo e que póe o trabalhador como um possível vetor de contaminação para sua família. Situações essas que acarretaram distanciamento dos familiares, medo, angústia e sentimentos de incerteza e estigmatização social ${ }^{2}$.

Nesse contexto, na medida em que a pandemia avança, os enfermeiros são atravessados por vivências que evocam sentimentos de luto. Luto é entendido como a perda de algo ou alguém importante; uma resposta a quebra de um vínculo significativo. Acredita-se que a maneira como a equipe de enfermagem vivencia o processo de luto na pandemia pode afetar sua saúde mental.

Os enfermeiros e técnicos têm vivenciado em seu cotidiano mortes mais frequentes do que aquelas com as quais estão acostumados a lidar, além da ausência ou modificação de rituais fúnebres. A ausência de rituais, além do distanciamento social acarretam um sentimento de solidão, sobrecarga de luto e exaustão emocional ${ }^{3}$. Uma resposta inadequada ao luto expõe os profissionais a um risco aumentado de sofrimento/transtornos mentais, luto persistente e um processo de luto prolongado ${ }^{4}$. Além disso, destaca-se o risco da chamada segunda onda da Covid-19 que se constitui de problemas de saúde psicológica, que nos profissionais de saúde se manifesta pelo entrelaçamento entre o cansaço e a sensação de desamparo 5 .

Diante disso, estudar as implicações das vivências de luto sobre a saúde mental da equipe de enfermagem é essencial para conduzir estratégias de cuidado e proteger os profissionais. Entre as diversas medidas de saúde pública diante de uma pandemia, a proteção psicoemocional dos profissionais da saúde é certamente uma das mais importantes.

Nesse momento em que as incertezas sobre a Covid-19 começam a diminuir e as recursos físicos e materiais de assistência à saúde estão mais organizados, emergem as preocupações com o ajustamento psicológico e a recuperação dos profissionais que se expõem físico-emocionalmente no enfretamento à Covid-196.

Diante do exposto, questiona-se qual o conhecimento científico produzido, até o momento, sobre os lutos vividos pelos profissionais de enfermagem em tempos de COVID-19? O objetivo desse estudo é mapear as evidências existentes na literatura sobre os lutos vividos pelos profissionais de enfermagem durante a pandemia da Covid-19 e discutir sobre as repercussões para sua saúde mental.

\section{MÉTODO}

Trata-se de um estudo do tipo revisão integrativa da literatura. A garantia do rigor científico e metodológico de estudo foram alicerçadas e adaptadas a partir das recomendações do Joanna Briggs Institute $(\mathrm{JBI})^{7}$ e do Preferred Reporting Items for Systematic reviews and Meta-Analyses ex- tension for Scoping Reviews (PRISMA-ScR). ${ }^{8}$ além um protocolo pré-estabelecido e na obediência das etapas da revisão: elaboração da pergunta de pesquisa; seleção da amostra, ou seja, estudos primários; extração dos dados; análise crítica dos estudos incluídos; síntese, interpretação e interpretação dos resultados da revisão; apresentação dos dados encontrados 9 .

A pergunta de pesquisa foi elaborada pela estratégia PICo que preconiza como elementos fundamentais: P - População; I- Interesse; e Co - Contexto. Assim, definiram-se os elementos: $\mathrm{P}$ - equipe de enfermagem ; I -vivências do luto ; e Co - COVID-19 ${ }^{10}$.

O estudo em questão tem como finalidade mapear as evidências existentes na literatura sobre os lutos vividos pelos profissionais de enfermagem durante a pandemia da Covid-19 e discutir sobre as repercussões para sua saúde mental.

Adotou-se como critérios de inclusão artigos disponíveis na íntegra, publicados entre os anos de 2019 e 2020, referentes à temática do estudo. O recorte temporal adotado é justificado pelo fato de 2019 ter sido o ano da descoberta do vírus Sars-cov-19, que poucos meses depois, viria a ser responsável pela referida pandemia. Os critérios de exclusão foram publicações duplicadas (manuscritos em duplicidade foram considerados apenas uma vez, sendo contabilizados na base de dados com maior número de referências (12). Não foi utilizado filtro de idioma.

A busca foi realizada em novembro de 2020 nas bases de dados Pubmed, Cinahl, Scopus e Lilacs, por meio dos descritores do vocabulário internacional utilizado na área da saúde - o Medical Subject Heading ( $\mathrm{MeSH})$ : Coronavirus Infections; bereavement; nursing; Mental Health, combinados pelo operador booleano AND.

A seleção dos estudos ocorreu seguindo três passos: leitura dos títulos e resumos e exclusão dos que não condisseram aos cri- 


\section{artigo}

Rocha, K.P.M.; Barros, W.C.T.S.; Oliveira, L.P.B.A.; Almeida-Júnior, J.J.A.;

Vivências de luto e saúde mental da enfermagem na pandemia da COVID-19: o que nos diz a literatura?

térios de elegibilidade; leitura na íntegra dos estudos pré-selecionados; elegibilidade dos estudos que se enquadraram nos critérios inclusão e compõem a amostra final do estudo ${ }^{11}$.

Após leitura dos estudos na integra, realizou-se a análise e descrição dos resultados obtidos. Os dados foram extraídos por um revisor, confirmados por um segundo revisor e as divergências resolvidas por consen- so entre os autores. Quanto ao tratamento dos dados, ressalta-se que as informações coletadas foram organizadas em um quadro sinóptico contendo título do artigo, objetivo, método, principais resultados e impressões dos revisores. Os achados foram analisados conforme a questão de pesquisa, apresentados por meio de quadros e sintetizados de forma narrativa.

Ressalta-se que estudos de revisão não

\section{Figura 1-Fluxograma do processo de identificação, seleção e inclusão dos estudos.}

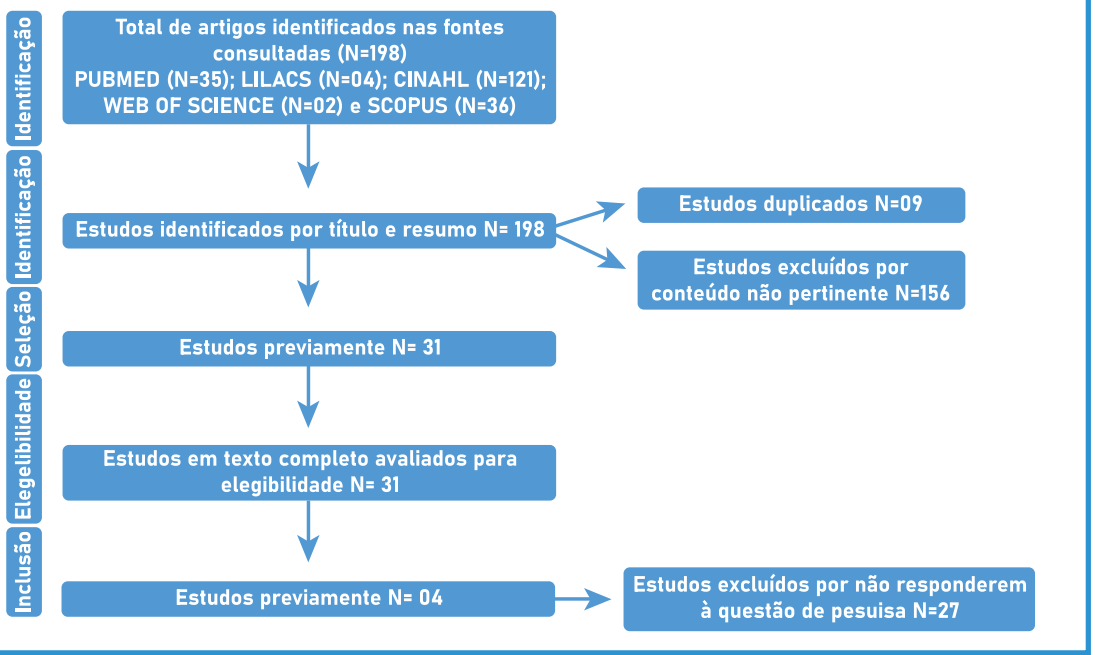

Quadro 1 - Síntese dos artigos selecionados sobre vivências de luto por profissionais de enfermagem durante a pandemia de covid-19.

\begin{tabular}{|c|c|c|c|c|}
\hline ESTUDO & TÍTULO DO ARTIGO & $\begin{array}{l}\text { PAIIS DE ORIGEM/ } \\
\text { PERIÓDICO }\end{array}$ & $\begin{array}{l}\text { OBJETIVOS/ DESENHO DE } \\
\text { ESTUDO }\end{array}$ & DESFECHO/CONCLUSÕES \\
\hline E1 & $\begin{array}{l}\text { Saúde mental dos } \\
\text { profissionais de saúde } \\
\text { na China durante } \\
\text { pandemia do novo } \\
\text { coronavírus: revisão } \\
\text { integrativa }\end{array}$ & Brasil & $\begin{array}{l}\text { Identificar as publicações relacio- } \\
\text { nadas com a saúde mental dos } \\
\text { profissionais de saúde atuantes } \\
\text { diante da pandemia de COVID-19 } \\
\text { revisão integrativa. }\end{array}$ & $\begin{array}{l}\text { Os textos trazem reflexões principal- } \\
\text { mente quanto a Insuficiência de treina- } \\
\text { mentos e equipamentos de proteção } \\
\text { individual no combate à COVID-19; } \\
\text { Sentimentos dos profissionais de saúde } \\
\text { no combate à COVID-19; Necessidade } \\
\text { de apoio psicológico/psiquiátrico aos } \\
\text { profissionais. }\end{array}$ \\
\hline E2 & $\begin{array}{l}\text { Fatores de estresse } \\
\text { nos profissionais } \\
\text { de enfermagem no } \\
\text { combate à pandemia } \\
\text { da COVID-19: síntese } \\
\text { de evidências }\end{array}$ & Brasil & $\begin{array}{l}\text { Identificar os principais efeitos } \\
\text { psicológicos da pandemia da } \\
\text { COVID-19 nos profissionais } \\
\text { de enfermagem; descrever os } \\
\text { principais fatores capazes de } \\
\text { gerar estresse psicológico nos } \\
\text { professionais de enfermagem; } \\
\text { descrever as estratégias de } \\
\text { coping para o combate ao } \\
\text { estresse emocional. } \\
\text { revisão da literatura }\end{array}$ & $\begin{array}{l}\text { o aumento da carga de trabalho, medo } \\
\text { de contaminar os familiares e de se } \\
\text { contaminar, desinformação e raiva do } \\
\text { governo e dos sistemas de saúde } \\
\text { são os principais fatores capazes } \\
\text { de gerar estresse emocional nos } \\
\text { profissionais de enfermagem. É mister } \\
\text { reconhecer e acolher os sentimentos } \\
\text { dos profissionais de enfermagem e } \\
\text { proporcionar uma esfera de estabilidade } \\
\text { em meio à crise }\end{array}$ \\
\hline
\end{tabular}




\begin{tabular}{|c|c|c|c|c|}
\hline E3 & $\begin{array}{l}\text { Saúde mental de } \\
\text { profissionais de } \\
\text { enfermagem durante } \\
\text { a pandemia de CO- } \\
\text { VID-19: recursos de } \\
\text { apoio }\end{array}$ & Brasil & $\begin{array}{l}\text { Refletir sobre as implicações da } \\
\text { pandemia de coronavirus na saúde } \\
\text { mental dos profissionais de enfer- } \\
\text { magem e os principais recursos de } \\
\text { apoio em desenvolvimento. } \\
\text { artigo reflexivo }\end{array}$ & $\begin{array}{c}\text { A experiência de trabalhar na pandemia } \\
\text { atual tem impactos físicos, emocionais, } \\
\text { econômicos, socias e psicológicos. É } \\
\text { importante entender e identificar reper- } \\
\text { cussões psicológicas e é preciso levar em } \\
\text { consideração as principais implicações } \\
\text { e emoções envolvidas antes, durante e } \\
\text { após o evento }\end{array}$ \\
\hline E4 & $\begin{array}{l}\text { Bereavement Support } \\
\text { on the Frontline of CO- } \\
\text { VID-19: Recommen- } \\
\text { dations for Hospital } \\
\text { Clinicians. }\end{array}$ & Reino Unido & $\begin{array}{l}\text { Revisar as evidências de pesquisa } \\
\text { relevantes e fornecer recomen- } \\
\text { dações baseadas em evidências } \\
\text { e recursos para os profissionais } \\
\text { de saúde mitigarem os resultados } \\
\text { ruins do luto e apoiar a equipe. } \\
\text { Revisão }\end{array}$ & $\begin{array}{l}\text { As recomendações incluem cuidado pré- } \\
\text { vio planejamento; comunicação proativa, } \\
\text { sensível e regular com os membros da } \\
\text { familia juntamente com o fornecimento } \\
\text { de informações precisas; habilitando } \\
\text { família membros para se despedirem } \\
\text { pessoalmente sempre que possível; su- } \\
\text { porte à comunicação virtual; fornecendo } \\
\text { excelente gerenciamento de sintomas e } \\
\text { emocionais e suporte espiritual; e forne- } \\
\text { cer e / ou sinalizar serviços de luto }\end{array}$ \\
\hline
\end{tabular}

Quadro 2 - Questões relacionadas às vivências de luto encontradas nos artigos selecionados.

\begin{tabular}{|c|c|}
\hline ESTUD0 & VIVÊNCIAS DE LUTO \\
\hline E1 & Alguns profissionais de cuidados intensivos se demitiram por falta de EPI ou condições de trabalho \\
\hline E2 & Perda de pessoas próximas; perda dos meios de subsistência; exclusão social por estar associado à doença. \\
\hline E3 & $\begin{array}{r}\text { Falta de contato com a familia } \\
\text { Pensamentos recorrentes sobre a pandemia e o processo de morte e morrer } \\
\text { Estigmas, medos e riscos de trabalhar com pacientes com } \\
\text { medo de adoecer e morrer }\end{array}$ \\
\hline E4 & interrupção das redes de apoio social dos parentes \\
\hline
\end{tabular}

\section{DISCUSSÃO}

A equipe de enfermagem, em seu processo de trabalho, é a que passa maior tempo na prestação de cuidados diretos aos pacientes com Covid-19. No entanto, esses cuidados não se restringem aos cuidados técnicos, mas envolvem seus aspectos psicológicos e emocionais de apoio ao paciente, ao mesmo tempo em que se vivência o medo de adoecer, de morrer e ainda da contaminação dos familiares e colegas $^{12}$.

Diversas situações podem influenciar na forma como o luto é vivido e administrado e, por consequência, contribuem tanto para o desenvolvimento do luto normal, quanto do luto complicado ou luto antecipatório. $\mathrm{O}$ luto antecipatório diz respeito à separação de um membro que se encontra em alta probabilidade de morte e assim elabora-se uma antecipação da perda. O luto prolongado perdura por mais de 6 meses e, nesse caso, o enlutado apresenta sinais intensos de tristeza, angústia de separação, perda e sentimentos de vazio ou falta de sentido de vida ${ }^{13}$.

Em situações típicas, $10 \%$ dos enlutados apresentam transtorno de luto prolongado. Outro fato que merece destaque é a possibilidade de estresse pós-traumático, no entanto, não foram encontrados estudos sobre sua incidência durante a pandemia de Covid-19.

Nos estudos analisados, as vivências do luto durante a pandemia da Covid-19 parecem estar permeadas por sentimentos de medo, desamparo, desesperança, sofrimento moral, estresse, ansiedade, sintomas depressivos, Burnout e impotência. É importante destacar que todos esses sentimentos estão atrelados aos fatores de risco de suicídio entre profissionais de enfermagem, um problema complexo, multidimensional e cada vez mais frequente em nosso meio e que, apesar de sua importância, segue silenciado no campo dos tabus das instituições ${ }^{14}$.
Os estudos trazem também algumas recomendações de proteção para a saúde mental dos profissionais de enfermagem. Essas dizem respeito ao apoio por teleatendimento em vários países do mundo, com destaque para as iniciativas nacionais do Conselho Federal de Enfermagem (COFEN) que dispõe de um grupo de enfermeiros especialistas em saúde mental para atender de forma gratuita e em tempo real colegas que necessitam de ajuda. Houve também no período entre maio e setembro um canal com psicólogos e psiquiatras disponíveis para teleconsulta, disponibilizado pelo Ministério da Saúde. Há também a recomendação de que o próprio trabalhador se monitore quanto a possíveis sinais e sintomas de agravamento de sua saúde mental ${ }^{12}$.

Nesse contexto, indica-se estratégias de autocuidado, gerenciamento de sinais e sintomas, suporte emocional e estratégias de resiliência. Por outro lado, destaca-se que essas estratégias não devem ser mais uma responsabilidade dos 


\section{artigo}

Rocha, K.P.M.; Barros, W.C.T.S.; Oliveira, L.P.B.A.; Almeida-Júnior, J.J.A.;

Vivências de luto e saúde mental da enfermagem na pandemia da COVID-19: o que nos diz a literatura?

profissionais que estão à frente dos cuidados, mas uma resposta organizacional que ofereça suporte emocional aos trabalhadores para que se possa prevenir e evitar sofrimentos ${ }^{12}$.

É necessário desenvolver estratégias que considerem todos esses fatores, especialmente a carga de trabalho por longas horas, ausência de descanso e aconselhamento psicológico, a fim de evitar o desgaste emocional dos trabalhadores ${ }^{16}$, além de acolher os sentimentos e gerar um clima de segurança e estabilidade apesar da situação de crise ${ }^{16}$.

Medidas importantes de cuidados como a adoção de instrumentos de rastreio especificamente sobre os impactos psicológicos da COVID-19 não foram citados pelos estudos ${ }^{15}$.

É pertinente compreender o luto como algo que se incorpora a existência humana e que é a partir das ressignificações das dores que os profissionais poderão melhorar emocionalmente suas vidas. Ressalta-se a necessidade do desenvolvimento de recursos para o auxílio aos profissionais durante a experiência do luto, que certamente trará marcas profundas nas pessoas que compõem a equipe de enfermagem ${ }^{17}$.

\section{CONCLUSÃO}

A presença de poucos artigos contidos na síntese final demonstra a lacuna existente no que concerne a vivência de luto pelos profissionais de enfermagem, o que por um lado explica-se pela contemporaneidade do tema e por outro lado pela compreensão ainda cercada de tabus sobre a necessidade de falar de morte, luto e saúde mental de enfermeiros e técnicos de enfermagem.

Os estudos aqui analisados foram de caráter descritivo, reflexivo ou de revisão, o que aponta a necessidade de novas pesquisas, em especial aquelas com desenhos experimentais e epidemiológicos, capazes de gerar maiores evidências científicas para fundamentar ações que ajudem no apoio aos profissionais no enfrentamento do luto durante a pandemia de Covid-19.

Acredita-se que este estudo contribuiu com o conhecimento do tema ao mesmo tempo em que lança luz sobre a necessidade de apoiar aspectos emocionais dos profissionais de enfermagem.

\section{REFERÊNCIAS}

1. Einboden, R. SuperNurse? Troubling the Hero Discourse in COVID Times. Health. 2020 Jul;24(4):343-7.

2. El-Hage, W; Hingray, C; Lemogne, C; Yrondi, A; Brunault, P; Bienvenu, T; eat all. Les professionnels de santé face à la pandémie de la maladie à coronavirus (COVID-19): quels risques pour leur santé mentale. Encephale. 2020 Jun;46(3):S73-S80.

3. Farahmandnia, B; Hamdanieh, L; Aghababaei, H. COVID-19 and Unfinished Mourning. Prehosp Disaster Med. 2020 Aug;35(4):464.

4. Pan, $H$. Deepening the understanding of complicated grief among Chinese older adults: A network approach. Asian J Psychiatr. 2020 Apr;50:101966.

5. Shojaei, S F; Masoumi, R. The Importance of Mental Health Training for Psychologists in COVID-19 Outbreak, Middle East J Rehabil Health Stud. Online ahead of Print; 7(2):e102846.

6. El-Hage, W; Hingray, C; Lemogne, C; Yrondi, A; Brunault, P; Bienvenu, $T$; eat all. Health professionals facing the coronavirus disease 2019 (COVID-19) pandemic: What are the mental health risks? 2020 Jun;46(3S):S73-S80.

7. Peters MD, Godfrey C, Mclnerney P, Munn Z, Tricco A, Khalil H. Chapter 11: Scoping Reviews. In: Aromataris E, Munn Z, Editors. BI Reviewer's Manual. JBI [Internet]. 2020 [acesso 2020 Nov 30]; Disponível: https:/dx.doi.org/10.7326/M18-0850

8. Tricco A, Lillie E, Zarin W, O'Brien $\mathrm{K}$, Colquhoun H, Levac D. PRISMA Extension for Scoping Reviews (PRISMA-ScR): Checklist and Explanation [Internet]. Annals of Internal Medicine. 2020 [acesso 2020 out 12]. Disponível em: https://doi.org/10.7326/M18-0850

9. Barra, DCC; Schuantes Paim, SMS; Dal Sasso, GTM; Colla, GW. MÉTODOS PARA DESENVOLVIMENTO DE APLICATIVOS MÓVEIS EM SAÚDE: REVISÃO INTEGRATIVA DA LITERATURA. Texto \& Contexto Enfermagem [Internet]. 2017;26(4):1-12. Recuperado de: https:/www. redalyc.org/articulo.oa?id=71453540029

10. Peters, MDJ; Godfrey, CM; Mclnerney, P; Soares, CB; Khalil, H; Parker,
D. The Joanna Briggs Institute reviewers' manual 2015: methodology for JBI scoping reviews [Internet]. 2015 [acesso 2020 Nov]. Disponivel em: http://joannabriggs.org/assets/docs/sumari/Reviewers-Manual_Methodologyfor-JBI-Scoping-Reviews_2015_v2.pdf

11. Rodrigues, TFCS; Cardoso, LCB; Rêgo, AS; Charlo, PB; Silva, M; Salci MA, Radovanovic, CAT. Exclusão social vivenciada por pessoas dependentes de drogas e seus familiares: revisão integrativa da literature. SaudColetiv (Barueri) [Internet]. 6 de agosto de 2020 [citado novembro de 2020];10(53):2506-19. Disponivel em: http://revistas.mpmcomunicacao.com.br/index.php/saudecoletiva/article/view/598

12. Ramos-Toescher, AM; Tomaschewisk-Barlem, JG; Barlem, ELD; Castanheira, JS; Toescher, RL. Saúde mental de profissionais de enfermagem durante a pandemia de COVID-19: recursos de apoio. Esc Anna Nery. 2020; 24(spe): 1-7.

13. Selman, LE; Chão, D; Sowden, R; Marshal, S. Chamberlain, C; Koffman, J. Bereavement Support on the Frontline of COVID-19:

Recommendations for Hospital Clinicians. Journal of Pain and Symptom Management. 2020; 60(2):81-86,

14. Freire, FO; Marcon, SR; Espinosa, MM; Santos, HGB; Kogien, M; Lima, NVP; Faria, JS. Fatores associados ao risco de suicídio entre enfermeiros e médicos: estudo transversal. Rev. Bras. Enferm. [online]. 2020; 73(1): 1-9.

15. Paiano, M; Jaques, AR; Nacamura, PAB; Salci, MA; Radovanovic, CAT; Carreira, L. Saúde mental dos profissionais de saúde na China durante pandemia do novo coronavírus: revisão integrativa. Rev. Bras. Enferm. 2020; 73(2): 1-9.

16. Barbosa, DJ; Gomes, MP; Souza, FBA; Gomes, AMT. Fatores de estresse nos profissionais de enfermagem no combate à pandemia da COVID-19: Síntese de Evidências. Com. Ciências Saúde. 2020; 31(1):31-47.

17. Freitas, JL. Luto, pathos e clínica: uma leitura fenomenológica. Psicol. USP. 2018; 29(1): 50-57. 